\title{
Mechanotendography in Achillodynia shows reduced oscillation variability of pre-loaded Achilles tendon: a pilot study
}

\author{
Laura Schaefer, Frank Bittmann \\ Regulative Physiology and Prevention, Department of Sport and Health Sciences, University of \\ Potsdam, Germany \\ This article is distributed under the terms of the Creative Commons Attribution Noncommercial License (CC BY-NC 4.0) which permits
} any noncommercial use, distribution, and reproduction in any medium, provided the original author(s) and source are credited.

\begin{abstract}
The present study focuses on an innovative approach in measuring the mechanical oscillations of pre-loaded Achilles tendon by using Mechanotendography (MTG) during application of a short yet powerful mechanical pressure impact. This was applied on the forefoot from the plantar side in direction of dorsiflexion, while the subject stood on the ball of the forefoot on one leg. Participants with Achilles tendinopathy (AT; $n=10$ ) were compared to healthy controls (Con; $n$ $=10$ ). Five trials were performed on each side of the body. For evaluation, two intervals after the impulse began $(0-100 \mathrm{~ms} ; 30-100 \mathrm{~ms})$ were cut from the MTG and pressure raw signals. The intrapersonal variability between the five trials in both intervals were evaluated using the arithmetic mean and coefficient of variation of the mean correlation (Spearman rank correlation) and the normalized averaged mean distances, respectively. The AT-group showed a significantly reduced variability in MTG compared to the Con-group (from $p=0.006$ to $\mathrm{p}=0.028$ for different parameters). The $95 \%$ confidence intervals (CI) of MTG results were disjoint, whereas the $95 \%$ CIs of the pressure signals were similar $(p=0.192$ to $\mathrm{p}=0.601)$. We suggest from this work that the variability of mechanical tendon oscillations could be an indicative parameter of an altered Achilles tendon functionality.
\end{abstract}

Key Words: Mechanotendography, mechanical tendinous oscillations, variability, impact on pre-activated Achilles tendon

Eur J Transl Myol 30 (2): 247-257, 2020

The etiology and pathomechanism of Achilles tendinopathy (AT) are still poorly understood, but seem to be multi-factorial. ${ }^{1,2}$ Achilles tendons withstand forces of $\sim 2600 \mathrm{~N}$ during walking and $\sim 9000 \mathrm{~N}$ during running. ${ }^{2}$ However, what causes the decline of its resilience? One of the most common hypotheses is the 'overuse' theory; where intuition dictates that Achilles function deteriorates due to excessive use during daily life. However, usually no extraordinary stress has been placed on the Achilles tendon prior to patient complaints. Of course, a correlation between complaints and physical strain is evident. ${ }^{3}$ There seems to be a disparity across patients with regards to individual development of tendon syndromes. Some may acquire a tendon syndrome under what is considered as a normal daily burden. Across athletes, not all of them show the same problems due to extreme loads. Why is this the case? The answer is clear: It is up to the relationship between load and personal resilience. In addition, it also has to be questioned, why patients frequently only develop complaints with one leg. Both legs carry the same load. In such cases, an overuse is unlikely. Therefore, it could be advantageous in the field of physiological medicine to consider factors that influence the resilience more closely. In therapy, unlike traditional orthopedic treatments, ${ }^{4}$ an eccentric exercise turned out to be efficient for treatment of AT. Reviews based on clinical trials have revealed high effectiveness of eccentric training. ${ }^{5}$ This also highlights the close connection between muscular functioning and AT. The Achilles tendon is a passive tissue, which links the triceps surae muscle to the calcaneus bone of the hindfoot. While the active neuromuscular part is rarely considered, there seems to be an altered neuromotor control in patients with AT. This is indicated by a strength reduction of plantar flexors. ${ }^{6}$ Some researchers have also found a decline in the amplitude of Electromyography (EMG), ${ }^{7}$ others have not. ${ }^{8}$ 
The mechanical muscular oscillations during muscular activity are well known, ${ }^{9}$ but have not been considered in the context of tendinopathy. Tendons show mechanical oscillations in frequency ranges $\sim 10 \mathrm{~Hz}$, which can be measured through the use of Mechanotendography (MTG). ${ }^{10-12}$ The MTG and the MMG of the associated muscle show coherent behavior. ${ }^{10,11}$ However, the tendinous oscillations are usually not examined. Martin et al. investigated the spread wave of the Achilles tendon during cyclic isometric ankle plantar flexion and walking. ${ }^{13,14}$ This is related to the present study, which focused on the mechanical oscillations of the Achilles tendon after a plantar impact on the pre-activated musculoskeletal system. This setting was chosen because related injuries often arise during physical activities characterized by impact absorption.

The purpose of this study was to investigate, whether or not the oscillatory behavior of the Achilles tendon shows differences between AT-patients and healthy controls. Standing on one forefoot is a setting, in which the subject continuously has to adapt to postural excursions, especially after perturbation. A high variability of neuromuscular control could be an advantage under these conditions.

\section{Materials and Methods}

The experimental study took place in the Neuromechanics Laboratory in the Division of Regulative Physiology and Prevention, at the University of Potsdam (Germany).

\section{Participants}

There were twenty healthy students ( $n=14$ male, $n=6$ female) who volunteered to be part of the Control (Con) group in this study. Exclusion criteria included: complaints of the lower extremities and spine within the last six months and pain on palpation of the Achilles tendon. Three participants had to be excluded because of complaints of the knee or ankle joint. The signals of another eight participants were excluded because of a technical phenomenon (see limitations). Accordingly, the signals of nine participants were included in the Con group. The average age, weight and height of this group was recorded. In the final Con group, there were eight males $(n=8: 22.75 \pm 1.87$ yrs., $79.19 \pm 7.3 \mathrm{~kg}, 184.5 \pm$ $6.75 \mathrm{~cm})$ and one female $(n=1: 22$ yrs., $60 \mathrm{~kg}, 165 \mathrm{~cm})$. There were also a group of patients with Achilles tendinopathy (AT) who volunteered to participate within the AT group $(n=23)$. Inclusion criteria were: complaints of the Achilles tendon and either a thickening of the Achilles tendon or a pain on palpation of at least 1 (pain scale: $0=$ no; $10=$ unbearable), or both. Exclusion criteria included: further complaints of the lower extremity or spine within the last six months or acute health problems, diabetes or hypo-/hyperthyroidism. Four patients were excluded on these grounds. Due to the signal phenomenon that also occurred in potential data from the provisional Con group, ten further patients were excluded for the same reason. The remaining patients formed the AT-group $(n=9)$ ). These were six males $(n=$ $6: 37.50 \pm 14.10$ yrs., $85.67 \pm 7.34 \mathrm{~kg}, 183.67 \pm 3.93 \mathrm{~cm})$ and three females $(n=3: 32.33 \pm 15.31$ yrs., $65 \pm 13.75$ $\mathrm{kg}, 170.33 \pm 9.07 \mathrm{~cm})$. Six patients had complaints on the left, three on both sides. The pain scale on palpation varied from 4 to 8 at different localizations of the tendon. There was also a test abortion criterion during measurement which was pain, yet that never occurred. The personal and anthropometric data (age, body mass,

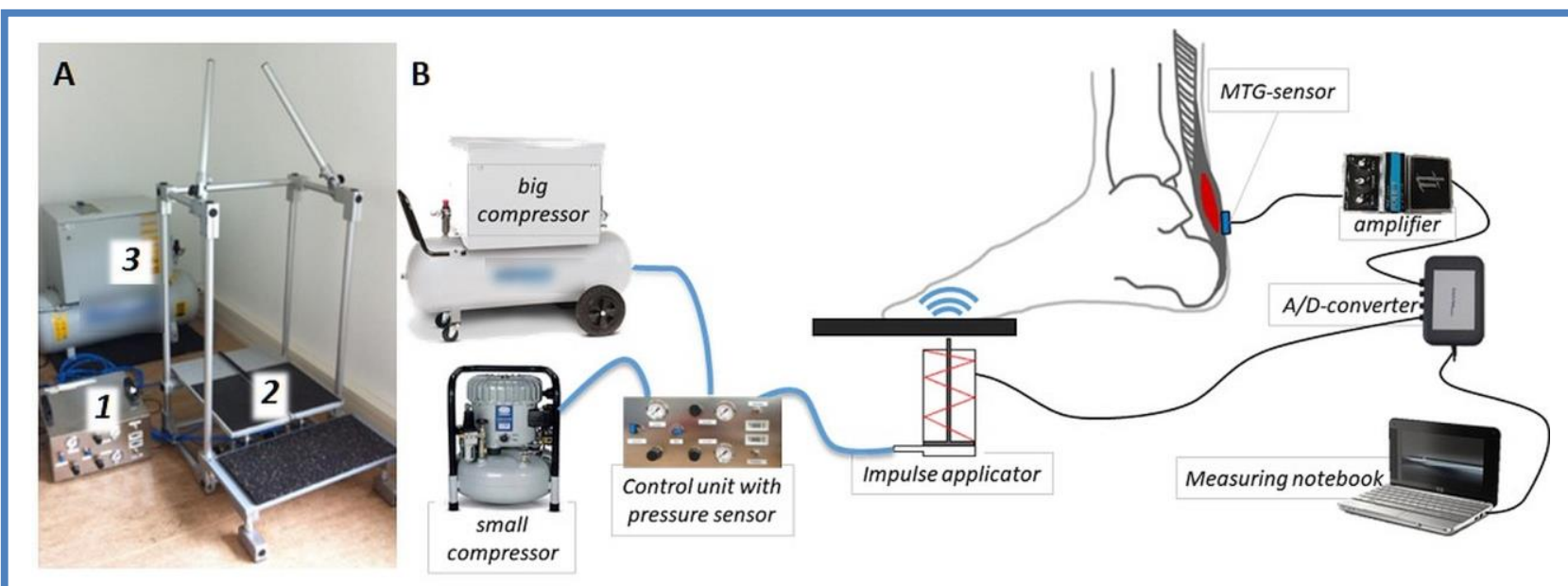

Fig 1. Setting. A, Impulse applicator with control unit (1), impulse panels (2) and compressor (3) for the application of the impulse on the lower extremities. $B$, The impulse applicator works with pressure and applies the impulse from plantar to the forefoot in upward direction. The thereby generated mechanical oscillations of the Achilles tendon are recorded with piezoelectric sensors (Mechanotendography; MTG). The analogue pressure and MTG-signals are converted (16-bit) to digital form, transferred to the measuring notebook and stored with Software DIAdem (National Instruments). 
body height) and health status (general acute or chronic diseases or other health issues, complaints of the musculoskeletal system) of all participants were assessed through a questionnaire and the Achilles tendon was examined clinically. The participants measurements were taken on both sides. Due to the technical phenomenon described earlier, the signals of only one side were utilized, except for one participant of each group. Therefore, the signals of each group consisted of $n=10$ usable MTG-signals. The study was approved by the local ethics committee at the University of Potsdam (Germany), approval no. 37/2015, and was conducted according to the Declaration of Helsinki. All subjects were informed in detail and gave written informed consent.

\section{Technical components of the setting}

In the set-up (see Figure 1) a strain of the pre-loaded Achilles tendon and its muscles was induced. The device produced a short but strong impact from the plantar part of the foot to the forefoot in an upward direction. This impulse generated a short dorsiflexion of the ankle and, thus, a stretch of the triceps surae system. In contrast to common reflex tests, this was performed during a preactivated muscle-tendon-system. By use of pneumatics, a fast, powerful, but short impulse was producible. The system, including the impulse platform, was constructed in collaboration with Seifert Drucklufttechnik $\mathrm{GmbH}$ (Bernsbach, Germany). The impulses were generated by two pneumatic cylinders (Riegler 7.DMA.80020 LINER, diameter $80 \mathrm{~mm}$ ) and transmitted by two separate panels (left and right side) to the plantar side of the forefoot. The main compressor provided the compressed air for the impulse with 8 bar (type Airko 3.0 HP, max. 10 bar). A second compressor (Jun-Air, Agre Compressor Hobby Star 200; max. 8 bar) was used to activate a pneumatically controlled valve for impulse pressure control. The impulse platforms were equipped with two pressure sensors (WIKA Type A-10, 0-10 bar; embedded in cylinders) and two one-axis ACC-sensors with a sensitivity of $312 \mathrm{mV} / \mathrm{g}$ (range $\pm 2 \mathrm{~g}$, linearity: $\pm 0.2 \%$; Biovision) for the detection of panel movements. The height and strength of pressure impulses were controlled by the control unit and the software DIAdem 10.2 (National Instruments (NI)). The impulse was adjusted by stroke height of $1 \mathrm{~cm}$ and duration of $0.02 \pm 0.002 \mathrm{~s}$. The technique of Mechanotendography (MTG) can be considered analogously to the Mechanomyography (MMG) technique, only applied for tendons. ${ }^{10-12}$ The established methods of MMG and EMG only provide information of muscular, not of tendinous structures. Due to the coherent behavior of MTG, MMG and EMG ${ }^{10,11,15,16}$ the MTG-signals allow indirect conclusions to be made regarding the muscular activity. Since it has been shown that the motion of the Achilles tendon and adjacent subcutaneous tissue behave similarly, ${ }^{13}$ we assume that the mechanical oscillations captured superficially reflect tendinous motions. During pilot testing, the following measurements were recorded: MMG of the gastrocnemius muscle and MTG of the Achilles tendon. Since the MMG did not provide further information concerning the variability, only the MTG was recorded at this stage. The oscillations of the Achilles tendon were recorded by a piezoelectric sensor (Shadow SH4001; amplifier: Nobels pre-amp booster pre-1). All signals (pressure, ACC, MTG) were transmitted through an A/D-converter (NI USB-6218, 16-bit) and recorded by NI DIAdem 10.2 (sampling rate: $500 \mathrm{~Hz}$ ).

\section{Reliability of the piezoelectric sensor}

In order to confirm reliability of the used sensor, the oscillations of a subwoofer were recorded by a piezoelectric sensor during playback of two different sounds. The sensor was fixed onto the coverage of the loudspeaker of the subwoofer (Yamaha Model No. NSSW210, Figure 2a). The signals were A/D converted (NI USB-6218, 16-bit) and recorded by NI DIAdem 14.0 software. Five repetitions were performed. The signals show high reproducibility as may be seen in Figure 2.
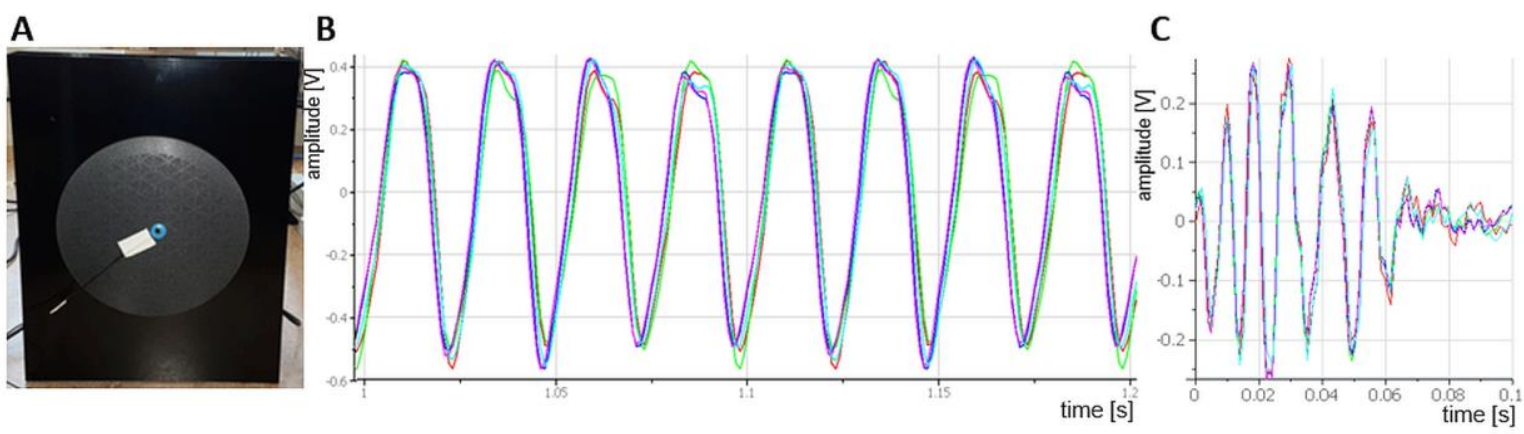

Fig 2. Reliability of the piezoelectric sensor used for the Mechanotendography (MTG). A, Setting for the audio measurements: piezoelectric sensor (pickup; Shadow SH4001) fixed on the cover of the loudspeaker of a subwoofer (Yamaha Model No. NS-SW210). B, Recorded $40 \mathrm{~Hz}$ sine oscillations (five repetitions, zoomed $0.2 s) . C$, Recorded oscillations of the audio from one MTG-signal from the Achilles tendon after impact (five repetitions, zoomed $0.1 \mathrm{~s}$ ). 

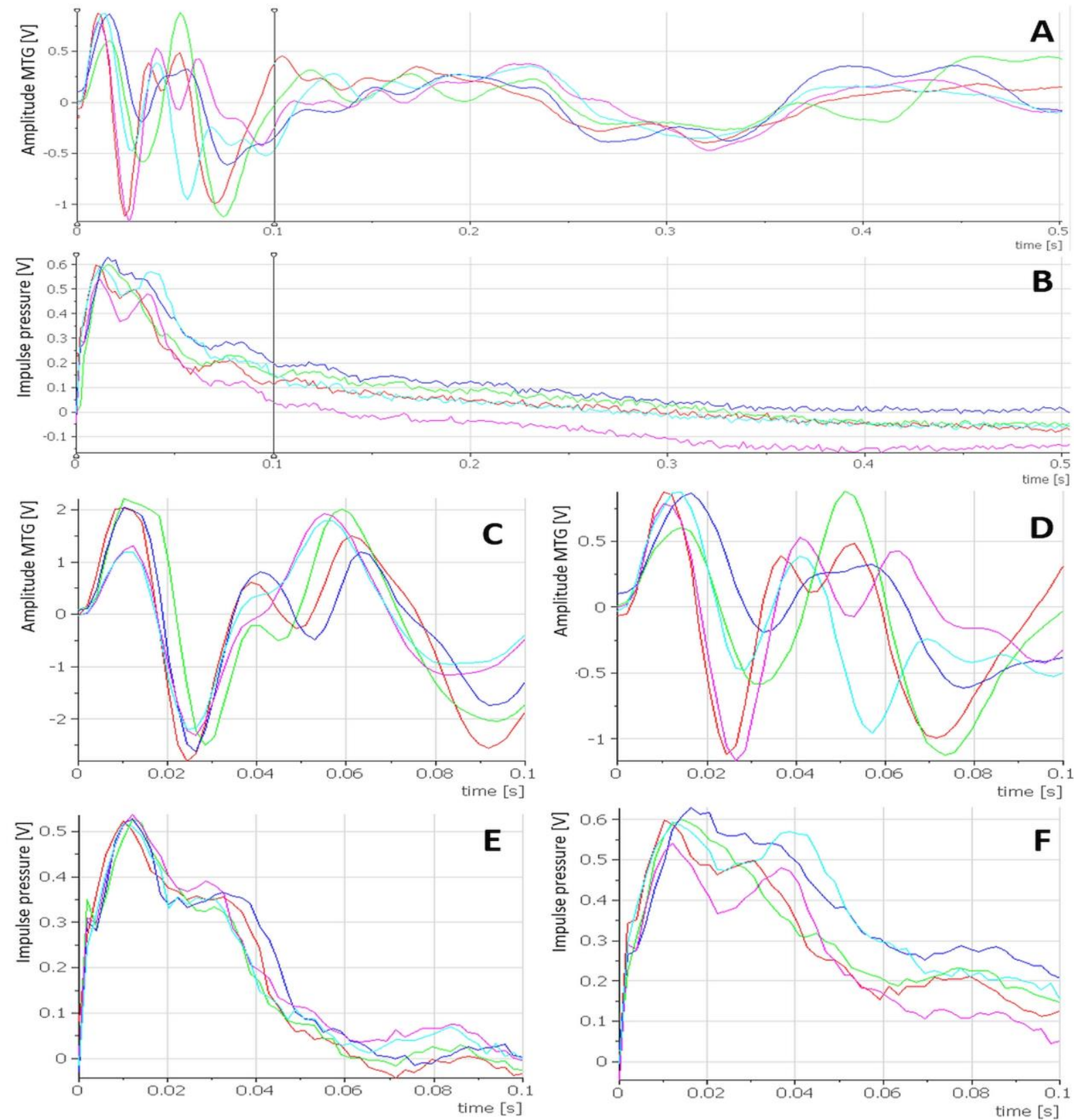

Fig 3. Exemplarily curves of MTG and pressure raw signals. Displayed are the 500-ms-intervals after impulse of the raw signals of the Mechanotendographie $(M T G)(A)$ and pressure signal (B) of each five trials of a healthy participant. For further analysis, the first 100-ms-interval after impulse starting is of special interest (vertical mark). The four diagrams below show exemplarily curves from impulse start to 100ms after impulse. Illustrated are each five MTG-signals of one patient with Achilles tendinopathy $(A T)(C)$ and of one control (Con) (D) as well as the corresponding five pressure signals of the AT-patient $(E)$ and the Con $(F)$.

Furthermore, the 40-Hz-oscillations are mapped precisely. From these measurements we therefore concluded that the piezoelectric sensor used was suited to measure oscillations in a reproducible way.

Setting and procedure for Achilles tendon measurements

The MTG-sensor was fixed $5 \mathrm{~mm}$ above the insertion of the Achilles tendon at the calcaneus using ECG-tape.
Participants stood habitually on one leg with the forefoot on the panel. The rear-foot was unloaded. The knee was bent slightly $\left(\sim 5^{\circ}\right.$ flexion, visually controlled $)$ to prevent the knee going into complete extension. In real-life motion, our knees are usually not completely extended, since this would diminish the absorbing properties of our musculoskeletal system. Therefore, our setting up of the Achilles tendon measurements was done so, keeping 
Table 1. Arithmetic mean (M), standard deviation (SD) and coefficient of variation (CV) of the ten Spearman correlation coefficients (MCC) of each participant of the MTG and pressure signals, respectively, of the $70 m$ s and 100ms-intervals after impulse. The significance ( $p)$ of the unpaired t-test or the Mann-Whitney$U$-test comparing Con vs. AT is also displayed.

\begin{tabular}{|c|c|c|c|c|c|c|c|c|}
\hline & \multicolumn{4}{|c|}{ 70ms-interval } & \multicolumn{4}{|c|}{$100 \mathrm{~ms}$-interval } \\
\hline & \multicolumn{2}{|c|}{ MTG } & \multicolumn{2}{|c|}{ pressure } & \multicolumn{2}{|c|}{ MTG } & \multicolumn{2}{|c|}{ pressure } \\
\hline & Con & AT & Con & AT & Con & AT & Con & $\mathrm{AT}$ \\
\hline 1 & $0.865 \pm 0.09$ & $0.844 \pm 0.15$ & $0.940 \pm 0.03$ & $0.592 \pm 0.26$ & $0.912 \pm 0.06$ & $0.885 \pm 0.08$ & $0.963 \pm 0.01$ & $0.968 \pm 0.02$ \\
\hline 2 & $0.288 \pm 0.40$ & $0.842 \pm 0.13$ & $0.895 \pm 0.05$ & $0.933 \pm 0.04$ & $0.469 \pm 0.19$ & $0.923 \pm 0.07$ & $0.915 \pm 0.03$ & $0.954 \pm 0.02$ \\
\hline 3 & $-0.125 \pm 0.74$ & $0.893 \pm 0.08$ & $0.904 \pm 0.45$ & $0.907 \pm 0.05$ & $-0.137 \pm 0.71$ & $0.760 \pm 0.11$ & $0.904 \pm 0.05$ & $0.943 \pm 0.04$ \\
\hline 4 & $0.140 \pm 0.70$ & $0.693 \pm 0.14$ & - & $0.948 \pm 0.03$ & $0.117 \pm 0.67$ & $0.832 \pm 0.08$ & - & $0.923 \pm 0.04$ \\
\hline 5 & $0.083 \pm 0.59$ & $0.814 \pm 0.12$ & - & $0.843 \pm 0.12$ & $0.250 \pm 0.43$ & $0.754 \pm 0.12$ & - & $0.929 \pm 0.04$ \\
\hline 6 & $0.008 \pm 0.57$ & $0.666 \pm 0.17$ & - & $0.864 \pm 0.07$ & $-0.074 \pm 0.63$ & $0.861 \pm 0.13$ & - & $0.823 \pm 0.11$ \\
\hline 7 & $0.457 \pm 0.31$ & $0.828 \pm 0.10$ & $0.902 \pm 0.07$ & $0.928 \pm 0.05$ & $0.567 \pm 0.26$ & $0.895 \pm 0.07$ & $0.932 \pm 0.04$ & $0.985 \pm 0.01$ \\
\hline 8 & $0.808 \pm 0.11$ & $0.977 \pm 0.02$ & $0.968 \pm 0.02$ & $0.781 \pm 0.10$ & $0.798 \pm 0.10$ & $0.955 \pm 0.03$ & $0.971 \pm 0.01$ & $0.914 \pm 0.04$ \\
\hline 9 & $0.819 \pm 0.11$ & $0.889 \pm 0.07$ & $0.897 \pm 0.08$ & $0.898 \pm 0.04$ & $0.887 \pm 0.07$ & $0.842 \pm 0.11$ & $0.925 \pm 0.05$ & $0.896 \pm 0.07$ \\
\hline 10 & $0.675 \pm 0.19$ & $0.744 \pm 0.17$ & $0.889 \pm 0.07$ & $0.918 \pm 0.04$ & $0.719 \pm 0.13$ & $0.745 \pm 0.17$ & $0.952 \pm 0.03$ & $0.918 \pm 0.04$ \\
\hline $\mathbf{M}$ & 0.402 & 0.827 & 0.939 & 0.861 & 0.451 & 0.845 & 0.938 & 0.925 \\
\hline SD & 0.372 & 0.097 & 0.048 & 0.107 & 0.392 & 0.073 & 0.022 & 0.045 \\
\hline $\mathbf{C V}$ & 0.926 & 0.117 & 0.051 & 0.124 & 0.869 & 0.087 & 0.023 & 0.048 \\
\hline $\mathbf{p}$ & \multicolumn{2}{|c|}{$.006 *$} & \multicolumn{2}{|c|}{0.601} & \multicolumn{2}{|c|}{ 0.011* } & \multicolumn{2}{|c|}{0.529} \\
\hline
\end{tabular}

*unpaired t-test with Welch-correction

$M T G=$ Mechanotendography $; A T=$ patients with Achilles tendinopathy; Con = controls

normal limb biomechanics in mind.The position on the panel was standardized in depth and width. In case subjects lost balance, struts were readily available so they could use them for support. A standing phase of at least $3 \mathrm{~s}$ before and after impulse had to be maintained. Five trials were done consecutively on each side. The side to start with was randomized in the Con group. The patients group (AT) started with the side with less, or no, complaints. The participants were instructed to stand on both legs and using their entire soles during the resting periods of $30 \mathrm{~s}$ between the trials.

\section{Data processing and statistical analysis}

The data analysis focused on the intrapersonal variability of the oscillations of the MTG-signals during the 100-ms interval after impact. Using the DIAdem software, two intervals were cut from raw data of all signals: a 100-ms interval (from the start of the pressure impulse) and a 70ms interval (from $30 \mathrm{~ms}$ after the impulse starting). The second interval was chosen since the oscillations in the first $30 \mathrm{~ms}$ seem to be rather similar. Within each interval, the following parameters were considered:

Correlation coefficient (CC)

The Spearman rank correlation coefficient was used to analyze the CC intrapersonally. This statistical analysis was performed using the statistics package IBM SPSS Statstics 25. The CC was calculated between two curves. Since five curves per sensor existed, ten CCs resulted for each sensor. The arithmetic mean (M), standard deviation (SD) and coefficient of variation (CV) of the ten CCs were calculated. One averaged CC (MCC) and its CV resulted per sensor.

Mean distances

The mean distance between each data point of each two curves was calculated. Since five curves per sensor exist, ten averaged mean distances resulted. These ten averaged mean distances were then normalized to the maximum amplitude of the signal and were averaged again. The resulting one value of normalized averaged mean distances (MD) was used for further analysis. The values of MCC and MD were checked for normal distribution 
Table 2. Displayed are the arithmetic mean and standard deviation of the ten normalized averaged distances (MD) of each participant of the MTG and pressure signals, respectively, of the 70ms and 100ms-intervals after impulse. The significance (p) of the unpaired t-test or the Mann-Whitney-U-test comparing Con vs. AT is also displayed.

\begin{tabular}{|c|c|c|c|c|c|c|c|c|}
\cline { 2 - 9 } \multicolumn{1}{c|}{} & \multicolumn{4}{c|}{ 70ms-interval } & \multicolumn{3}{c|}{ 100ms-interval } \\
\cline { 2 - 10 } \multicolumn{1}{c|}{ MTG } & \multicolumn{2}{c|}{ Con } & AT & Con & AT & Con & AT & \multicolumn{2}{c|}{ Con } & AT \\
\hline 1 & $0.089 \pm 0.04$ & $0.158 \pm 0.08$ & $0.051 \pm 0.03$ & $0.074 \pm 0.03$ & $0.077 \pm 0.03$ & $0.144 \pm 0.07$ & $0.056 \pm 0.03$ & $0.090 \pm 0.03$ \\
\hline 2 & $0.255 \pm 0.07$ & $0.133 \pm 0.06$ & $0.140 \pm 0.06$ & $0.063 \pm 0.04$ & $0.243 \pm 0.04$ & $0.120 \pm 0.05$ & $0.130 \pm 0.05$ & $0.073 \pm 0.04$ \\
\hline 3 & $0.406 \pm 0.23$ & $0.095 \pm 0.04$ & $0.154 \pm 0.07$ & $0.129 \pm 0.07$ & $0.411 \pm 0.25$ & $0.085 \pm 0.04$ & $0.152 \pm 0.07$ & $0.121 \pm 0.06$ \\
\hline 4 & $0.426 \pm 0.24$ & $0.193 \pm 0.07$ & - & $0.083 \pm 0.05$ & $0.401 \pm 0.23$ & $0.174 \pm 0.05$ & - & $0.076 \pm 0.03$ \\
\hline 5 & $0.282 \pm 0.18$ & $0.144 \pm 0.05$ & - & $0.064 \pm 0.02$ & $0.261 \pm 0.15$ & $0.138 \pm 0.04$ & - & $0.061 \pm 0.01$ \\
\hline 6 & $0.311 \pm 0.13$ & $0.136 \pm 0.04$ & - & $0.035 \pm 0.01$ & $0.309 \pm 0.12$ & $0.127 \pm 0.03$ & - & $0.052 \pm 0.02$ \\
\hline 7 & $0.187 \pm 0.08$ & $0.125 \pm 0.03$ & $0.070 \pm 0.03$ & $0.102 \pm 0.06$ & $0.163 \pm 0.07$ & $0.118 \pm 0.03$ & $0.078 \pm 0.02$ & $0.097 \pm 0.05$ \\
\hline 8 & $0.118 \pm 0.03$ & $0.114 \pm 0.06$ & $0.076 \pm 0.04$ & $0.049 \pm 0.02$ & $0.130 \pm 0.03$ & $0.095 \pm 0.04$ & $0.084 \pm 0.04$ & $0.063 \pm 0.02$ \\
\hline 9 & $0.129 \pm 0.04$ & $0.105 \pm 0.03$ & $0.076 \pm 0.06$ & $0.044 \pm 0.01$ & $0.110 \pm 0.03$ & $0.111 \pm 0.04$ & $0.070 \pm 0.04$ & $0.066 \pm 0.02$ \\
\hline 10 & $0.181 \pm 0.06$ & $0.176 \pm 0.05$ & $0.069 \pm 0.03$ & $0.056 \pm 0.03$ & $0.164 \pm 0.05$ & $0.169 \pm 0.05$ & $0.068 \pm 0.03$ & $0.073 \pm 0.03$ \\
\hline M & 0.238 & 0.138 & 0.091 & 0.070 & 0.227 & 0.128 & 0.091 & 0.077 \\
\hline SD & 0.118 & 0.031 & 0.039 & 0.029 & 0.118 & 0.029 & 0.036 & 0.020 \\
\hline CV & 0.495 & 0.223 & 0.433 & 0.407 & 0.521 & 0.225 & 0.392 & 0.263 \\
\hline $\mathbf{p}$ & & $\mathbf{0 2 6}$ & & 0.192 & & $\mathbf{0 . 0 2 8} *$ & 0.321 \\
\hline
\end{tabular}

*unpaired t-test with Welch-correction

$M T G=$ Mechanotendography $; A T=$ patients with Achilles tendinopathy $;$ Con = controls

by using a Shapiro-Wilk test. The Mann-Whitney-U-test or the t-test for independent samples and ANOVA (including the factors of: gender, age and BMI) were performed for group comparisons. If the homogeneity of variance (Levène test) was not fulfilled, the Welch correction was used $\left(\mathrm{p}_{\mathrm{adj}}\right)$. The effect size was calculated by using Pearson's correlation coefficient $(r)$ or by partial eta-squared $\left(\eta^{2}\right)$. The $95 \%$ confidence interval (CI) of each parameter was calculated. Significance level was set at $\alpha=0.05$.

\section{Results}

Curve characteristics

Each five trials of raw data (MTG, pressure) of the 500ms interval after impact of one control and of the 100-ms intervals of one AT and one Con, are displayed in Figure 3. In the MTG signal, clear oscillations of high magnitude with a period duration of $\sim 30 \mathrm{~ms}$ were visible immediately after impulse. After $100 \mathrm{~ms}$, the magnitude decreased, whereas an additional oscillation with $\sim 230 \mathrm{~ms}$ period appeared.

\section{Correlation of curves}

In both intervals, the MCC of pressure was similar between Con and AT (100ms: $\mathrm{t}(15)=-0.645 ; p=0.529$, $r=0.164$; 70ms: $U=29.00, p=0.601)$, whereas the MCC of MTG was significantly higher in AT $(100 \mathrm{~ms}: t(18)=$ 3.138; $p_{a d j}=0.011, r=0.60 ; 70 \mathrm{~ms}: t(10.172)=-3.434$, $\left.p_{a d j}=0.006, r=0.73\right)$. These data are seen in Table 1 .

The $95 \%$ CI of MCC of pressure and MTG-signals for both groups are shown in Figure 4. The latter were disjoint, which reflects high significance, whereas the CIs of MCC of pressure signals showed similar endpoints.

Since AT and Con differed significantly in age $(t(15)=2.807 ; p=0.022)$, the group differences have to be questioned. Including only participants between 20-40 years of age (AT: $n=6$; Con: $n=10$; age comparison: $t(12)=2.033 ; p=0.087)$, the correlation of the MTGsignals still differed significantly (MTG: $t(14)=3.032$; $p_{\text {adj }}=.013, r=0.63$; pressure: $t(13)=-0.413 ; p=0.686$, $r=0.11)$. The complete model of multifactorial ANOVA 


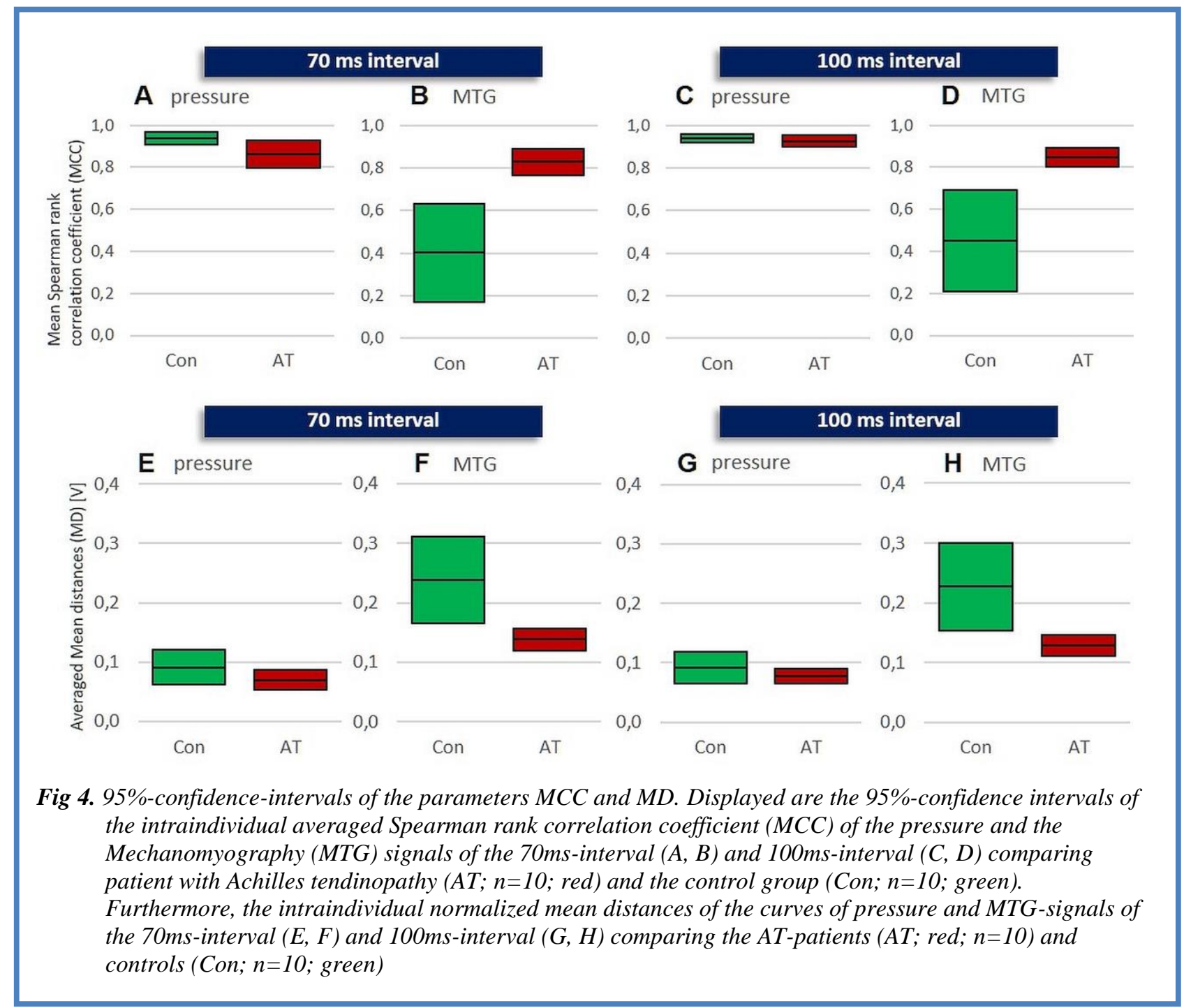

regarding the MCC of MTG was found to be statistically significant $\left(F(8,11)=6.172, p=0.004, \eta^{2}=0.82, R_{\text {corr }}^{2}=\right.$ $0.69, n=20)$. Thereby, the MCC of MTG depended significantly on the diagnosis of Achillodynia $\left(F(1,11)=29.88, p=0.000, \eta^{2}=0.731\right)$. The gender, BMI and age were found to have had no significant effects $(p=0.053-0.503)$. Furthermore, there was a significant interaction of diagnosis and gender $\left(F(1,11)=5.749, p=0.035, \eta^{2}=0.343\right)$ as well as of diagnosis and BMI $\left(F(1,11)=7.962, p=0.017, \eta^{2}=\right.$ $0.420)$. Thereby, the MCC of male was found to be higher compared to female (m: $0.86 \pm 0.08$; f: $0.81 \pm 0.05)$. The within subject CVs of CC of the AT-group amounted to $11.7 \pm 5.6 \%$ compared to the controls with $48.8 \pm$ $328.2 \%$. The pressure signals showed similar withinsubject CVs for both groups (AT: $4.9 \pm 3.6 \%$; Con: $3.6 \pm$ $1.8 \%)$.

Mean distances of curves

The MDs of MTG were significantly higher in Con vs. AT for both intervals $\left(100 \mathrm{~ms}: t(10.075)=2.566, p_{a d j}=\right.$ $0.028, r=0.629$; 70ms: $t(10.231)=2.604, p_{a d j}=0.026$, $r=0.631$ ), whereas the MDs of pressure signals (Table 2) did not differ significantly (100ms: $U=21, p=0.193$; 70ms: $t(15)=1.027, p=0.321)$. The $95 \%$ CIs displayed the higher variance of the MDs concerning the MTGsignals in Con vs. AT group, whereas the MDs of the pressure signals showed similar 95\% CIs (Figure 4).

\section{Discussion}

The results presented support the hypothesis that patients with AT show changed oscillatory patterns of the Achilles tendon in the form of a lower variability compared to controls. This was demonstrated by the significantly higher correlation and the significantly lower normalized mean distances of the MTG-signals in the first $100 \mathrm{~ms}$ after impact during repeated trials in ATpatients. This leads to the assumption that the variability of tendinous oscillations is reduced in affected tendons. The within-subject CV of CC supports this assumption. We found a CV of $\sim 50 \%$ in controls and $\sim 12 \%$ in ATpatients, whereas the CVs of pressure signals were similar with $4 \%$ and $5 \%$, respectively. 


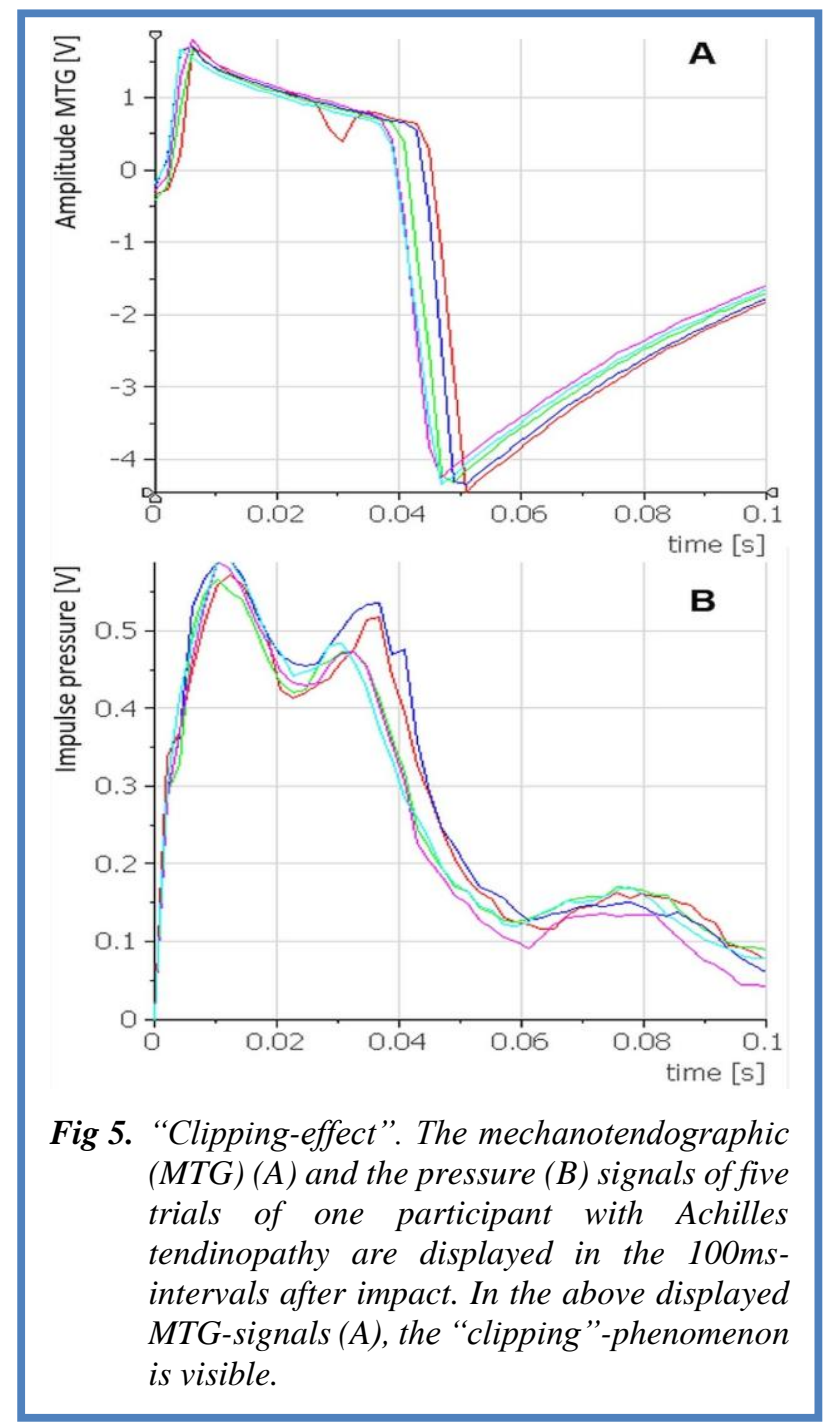

\section{Methodological limitations}

The methods of MMG and MTG are commonly applied in our laboratory. However, a technical phenomenon, known as the clipping effect (Figure 5). has never occurred before. The curve shape is atypical for biological data. The authors interpret this as a technical artefact. Impulses with substantially large amplitudes lead likely to a "clipping effect" caused by the amplifier. In audio signal processing, this phenomenon occurs when an amplifier is overdriven. The extreme excursions of the tendon immediately after impact could be the cause of overdriving in our experiments. A reduction in gain would solve this problem.

Despite this, our results are usable groundwork and sufficient for the pilot nature of the study performed. The phenomenon should not have influenced the validity of the results. However, the exclusion of those signals lead to a smaller sample size, which reduced the quality.

In the following content-related discussion, the results have to be interpreted with caution due to the pilot character. Nevertheless, they suggest a reduction in variability of the mechanical oscillations of the Achilles tendon in Achillodynia.

\section{MTG as simple and promising method}

Tendons function as a passive strand of connective tissue, which is driven by active elements - the muscles. When active, muscles tighten the sinew. In this process, muscles produce stochastically distributed mechanical oscillations. $^{10,11}$ The oscillations work in an axial direction along the tendinous thread. However, muscles also vibrate laterally. Consequently, they act as active oscillators stimulating the passive tendon longitudinally and laterally. The oscillations of collaborating muscles can be synchronized by the neuromuscular system. ${ }^{10,11} \mathrm{~A}$ specific feature of the Achilles tendon is that three muscle heads drive the tendon. It remains undetermined how exactly these three oscillating actuators cooperate. In any case, a superpositioning effect on the tendon can be expected.

With regards to the muscle-sinew system that was the focus of this paper, the triceps surae system is pre-loaded isometrically. At this stage, the impulse was set and tightened the sinew intensely. The behavior of the tendinous string during and after this impact is influenced by the active drives mentioned. However, the passive mechanical properties also play a role. The resonance frequency depends on the tension and length. Consequently, the tendon works as a bandpass and certain frequencies are suppressed. Furthermore, the vibration damping of the surrounding soft tissues has to be considered. Therefore, it is supposed that the tendinous oscillations do not simply represent the muscular vibrations. Rather, the tendinous behavior is highly influenced by the tension and oscillations of the attached muscles. We suggest that the tendinous oscillations may provide insights into neuromuscular control and perhaps also provide further insights into the pathomechanism of AT. Structural changes such as thickening or edemas of the tendon are often observed in AT. In addition, impaired muscle function is likely. ${ }^{17}$ Since all these factors influence the mechanical properties of the tendon, MTG could be a useful approach in the diagnosis of AT.

\section{Curve shape}

The curve shapes of the MTG show a rising magnitude with its maximum after $\sim 10 \mathrm{~ms}$ post-impact. This complies with the increase of pressure impulse. Therefore, it is interpreted as a result of the initial tightening of the tendon caused by the dorsiflexion. The second, negative swing is interpreted as countermovement of the tendon. The impact was standardized; consequently, all signals show a nearly stereotypical curve shape in the first $30 \mathrm{~ms}$. Afterwards, the tendinous oscillations seemed to vary in a wide range. This phase might show, how the neuromuscular system manages perturbation in order to restore balance. Physiologically, the impact must have an effect on the muscle spindles due 
to the tightening of the tendon. This might initiate the short latency reflex. ${ }^{18}$ On the assumption that a segmental stretch reflex is triggered by the impact, the response of the nervous system can be expected after $\sim 30 \mathrm{~ms}$. This is in agreement with other described electrophysiological data that have shown an ankle tendon reflex of $\sim 35 \mathrm{~ms} .{ }^{19}$ Other researchers describe longer latencies of the triceps surae stretch reflex of $\sim 49 \mathrm{~ms}$ or $\sim 55-65 \mathrm{~ms}^{20}$ Thereby, the setting included a moveable platform, on which the subjects stood. This would rather correspond to the presented setting. The former investigation used a reflex hammer, which probably caused different reflex latencies. Additionally to the segmental electrophysiological reply, the muscles most likely require $20-30 \mathrm{~ms}$ longer to create the mechanical answer. ${ }^{21}$ This is based on current knowledge of reflex measurements in relaxed muscles. An electromechanical delay between EMG and MMG of $\sim 11.1 \pm 0.5 \mathrm{~ms}$ and a delay between MMG and force response of $\sim 13.0 \pm$ $1.3 \mathrm{~ms}$ have been reported. ${ }^{22}$ In total, the EMG-forcedelay amounts to $\sim 23-24 \mathrm{~ms} .^{23}$ Esposito et al. reported latencies of $45 \mathrm{~ms}$ from EMG to force response and only $\sim 3$ ms delay from EMG to MMG. ${ }^{24}$ The different results described could have been due to variable settings. There is only minimal experience across the literature with the reflex behavior of pre-activated muscles, as shown here. Thereby, the nervous motor pathways must be at a higher operation level. Priming effects are conceivable. In active muscles, the response threshold is reduced by 2 to $6 \mathrm{~ms}$ compared to relaxed muscles using cortical stimulation. ${ }^{25}$ Forgaard et al. postulate that perturbation can trigger a voluntary response at short latencies lower than $100 \mathrm{~ms}$ in upper limbs. ${ }^{26} \mathrm{We}$ assume that a voluntary response of lower limbs might last longer. Nevertheless, it would be conceivable that a voluntary response within the first $100 \mathrm{~ms}$ could influence the presented curve shape. After $100 \mathrm{~ms}$, the curve shape begins to return to regular oscillatory behavior. This is interpreted as a sign of voluntary neuromuscular control in order to maintain balance. Forgaard showed that the intention to respond to perturbations increases the long-loop reflex (M2). ${ }^{26}$ Since in the present investigation the participants maintained balance after the impact, we suppose that the M2 could be larger than without this task. We assume multifactorial causes that would explain the MTG curve shape after impact. These could range from clearly mechanical causes, up to supraspinal mechanisms.

\section{Variability as a possible indicator of neuromusculo-} skeletal diseases

The presented results showed that there was a significantly lower variability in AT-patients compared to controls. This supports the assumption that a certain percentage of variability seems to be a sign of proper function. Stam et al. ${ }^{27}$ detected a within subject variability of the peak-to-peak-amplitude in EMG of $\sim 18 \%$ after reflex stimulus of the soleus tendon in healthy participants, for example which highlights the magnitude of this variability. The ground reaction force during human walking is a frequently used parameter. Studies have indicated that there is a non-pathological withinsubject variability of $7 \%$ (vertical) and of $20 \%$ (horizontal). ${ }^{28}$ This supports the assumption some variability should be present in healthy neuromuscular systems. Variability may also arise due to sideasymmetry, which has been the focus of other investigations. It would be expected that in healthy subjects a non-significant side-asymmetry is present. In a study by Jelen and colleagues, 77 able-bodied subjects showed a side-asymmetry of $12-16 \%$ in gait lines, which was considered as non-pathological. ${ }^{29}$ Five patients with herniated lumbar disc showed a greater asymmetry of $\sim 20-55 \%$. This idea has been further supported by the work of Bittman and co-workers who used gait-lineanalyses. The authors found that side-asymmetry increased up to $28-57 \%$ in patients with lower limb complaints. $^{30}$ Thereby, the affected side showed a reduced variability. These results support the present study, since the oscillations were found to be less variable in AT-patients. We consider the variability as an indicator for the adaptability of the neuromuscular system. The lower variability in tendinous oscillations after impact is interpreted as a sign of pathological behavior. The measurements were performed under pathological conditions for the group with AT. In a number of cases, the sinew was thickened or edema was present in the surrounding connective tissue. As mentioned above, these alterations of passive structures might affect the oscillations. Although it seems to be unlikely that this would reduce the variability of oscillations, an influence cannot be ruled out completely. The mentioned pathological conditions include not only structural alterations but also nociceptive afferents. This might affect the motor control. During walking and standing unilaterally on the forefoot, the correct processing of the complex proprioceptive afferents is essential to keep balance after perturbations. Supposing that the afferent stream is affected at the spinal level, the supraspinal nervous system will be restricted in regulating the postural equilibrium during gait or stance. According to the Gate Control Theory, a certain level of nociceptive signals could influence the afferent flow of proprioceptive signals. ${ }^{31}$ Consequently, only a basic program of motor control would probably be executed, which could limit the adequate adaptation to instantaneous requirements. This could lead to stereotypical behavior and a loss of variability. An impaired adaptability is possibly characterized by a reduction in variability of those non-stationary stochastically distributed oscillations. Eventually the question arises, if the tendinous complaints and alterations could be caused by the stereotypical mechanical behavior. It would be conceivable that a frequently repeated similar strain of the muscle-tendoncomplex during gait could lead to a monotonous stress to the fibrous tissue. This could possibly entail a structural 
modification of the tendon. In conclusion, the findings of our study support previous studies that indicate a lower variability of neuromuscular parameters in patients with complaints. It remains unclear, if the changed oscillatory behavior is caused by the complaints or vice versa. However, it likely indicates an impairment of neuromuscular control, which is necessary to react and adapt to external forces and might lead to musculoskeletal complaints. Further investigations could confirm the results of our pilot study. This would provide a comprehensive basis for extensive interpretation and transmission into practice. Studies should also examine if the reduced variability is apparent prior to structural changes or if symptom-free thickened tendons show changes in variability. The question of causation remains unanswered. If the results will be supported by further studies, the correlation technique of MTG-signals after impact could be applicable in diagnostics of musculoskeletal pathologies such as Achilles tendinopathy.

\section{List of acronyms}

AT - Achilles tendinopathy group

ACC - acceleration

CI - confidence interval

Con - control group

$\mathrm{CV}$ - coefficient of variation

EMG - Electromyography

$\mathrm{M}$ - arithmetic mean

MCC - mean correlation coefficient

MD - mean distances

MMG - Mechanomyography

MTG - Mechanotendography

MVIC - maximal voluntary isometric contraction

NI DIAdem ${ }^{\mathrm{TM}}$ - National Instruments DIAdem ${ }^{\mathrm{TM}}$

SD - standard deviation

\section{Authors contributions}

FB and LS contributed to design the study. LS and FB performed the acquisition, LS the measuring and analysis of data. In the process of interpretation and discussion of data, LS and FB participated. LS drafted the manuscript and it was critically revised by FB and LS. All authors contributed to manuscript revision, and read and approved the submitted version.

\section{Acknowledgments}

The authors would like to thank all subjects for participating in the study and for the interest in our investigations. We acknowledge the support of Deutsche Forschungsgemeinschaft (German Research Foundation) and Open Access Publication Fund of Potsdam University.

\section{Funding}

The study (construction of the pneumatic impulse system) was funded by the Federal Ministry of Economics and Technology (ZIM KF-2262306KJ1).

\section{Conflict of Interest}

The authors have no conflicts to disclose

\section{Ethical Publication Statement}

We confirm that we have read the Journal's position on issues involved in ethical publication and affirm that this report is consistent with those guidelines.

\section{Corresponding Author}

Dr. Laura Schaefer, Regulative Physiology and Prevention, Department Sports and Health Sciences, Human Sciences Faculty, University of Potsdam, KarlLiebknecht-Str. 24-25, building 24, room 1.14, 14476 Potsdam, Germany. Phone: +49.331.9772987, Fax: + 49.331.9772731. ORCID iD: 0000-0002-6289-6987

E-mail: 1schaefe@uni-potsdam.de

E-mail and ORCID iD of co-author

Frank Bittmann: bittmann@uni-potsdam.de ORCID iD: 0000-0003-4514-8060

\section{References}

1. Ackermann PW, Phisitkul P, Pearce CJ. Achilles tendinopathy - pathophysiology: state of the art. Journal of ISAKOS: Joint Disorders \& Orthopaedic Sports Medicine 2018;3:304-14. doi: 10.1136/jisa kos-2017-000164.

2. Hirschmüller A. Achillodynie - Pathophysiologie und Diagnostik. Manuelle Therapie 2014;18:107-12. DOI 10.1055/s-0034-1383426

3. Järvinen TAH, Kannus P, Paavola M et al. Achilles tendon injuries. Current Opinion in Rheumatology 2001;13:150-5. ISSN 1040-8711

4. Kearney RS, Parsons N, Metcalfe D, Costa ML. Injection therapies for Achilles tendinopathy. Cochrane Database of Systematic Reviews 2015;5:CD010960. DOI: 10.1002/14651858.CD010 960.pub2

5. Krämer R, Lorenzen J, Vogt PM, Knobloch K. Systematische Literaturanalyse über exzentrisches Training bei chronischer Mid-portionAchillestendinopathie: Gibt es einen Standard? Systematic Review about Eccentric Training in Chronic Achilles Tendinopathy. Sportverletz Sportschaden 2010;24:204-11. DOI: $10.1055 / \mathrm{s}-$ 0029-1245820;

6. Mahieu NN, Witvrouw E, Stevens E et al. Intrinsic risk factors for the development of Achilles tendon overuse injury - a prospective study. The American Journal of Sports Medicine 2006;34(2). DOI: 10.1177/0363546505279918

7. Baur H, Divert C, Hirschmuller A et al. Analysis of gait differences in healthy runners and runners with chronic Achilles tendon complaints. Isokinet Exerc Sci 2004;12:111-6. DOI: 10.3233/IES-2004-0161

8. Baur H, Müller S, Hirschmüller A et al. Comparison in lower leg neuromuscular activity between runners with unilateral mid-portion Achilles tendinopathy and healthy individuals. Journal of 
Electromyography and Kinesiology 2011;21:499505 https://doi.org/10.1016/j.jelekin.2010.11.010.

9. Beck T. Applications of Mechanomyography for Examining Muscle Function. Editor Travis W Beck. India: Transworld Research Network; 2010. ISBN 978-81-7895-449-3

10. Schaefer L, Bittmann F. Coherent behavior of neuromuscular oscillations between isometrically interacting subjects: experimental study utilizing wavelet coherence analysis of mechanomyographic and mechanotendographic signals. Scientific Reports 2018;8:15456. DOI:10.1038/s41598-01833579-5. https://rdcu.be/9wm2

11. Schaefer LV, Torick AH, Matuschek $\mathrm{H}$ et al. Synchronization of muscular oscillations between two subjects during isometric interaction. Eur J Trans Myol 2014;24(3):195-202. DOI 10.4081/ejtm. 2014.2237

12. Torick A, Hoff M, Schaefer $L$ et al. Mechanotendografie (MTG) - Messen und Analysieren der Oszillationsmuster von Achillessehnen. Poster presentation at the 44th Congress of German Medicine 2013 in Frankfurt am Main, Germany. Deutsche Zeitschrift für Sportmedizin 2013;64(7-8):206.

13. Martin JA, Brandon SCE, Keuler EM et al. Gauging force by tapping tendons. Nature communications 2018;9:1592. DOI: 10.1038/s41467-018-03797-6.

14. Keuler EM, Loegering IF, Martin JA et al. Shear wave predictions of Achilles tendon loading during human walking. Scientific Reports 2019;9:13419. https://doi.org/10.1038/s41598-019-49063-7

15. Beck TW, Housh T, Fry AC et al. MMG-EMG Cross Spectrum and muscle fiber type. Int J Sports Med 2009;30(7):538-44. DOI: 10.1055/s-0029-1202349

16. Yoshitake Y, Shinohara M, Ue H, Moritani T. Characteristics of surface mechanomyogram are dependent on development of fusion of motor units in humans. J Appl Physiol 2002;93:1744-52. https://doi.org/10.1152/japplphysiol.00008.2002

17. Segeser B, Goesele A, Renggli P. The Achilles tendon in sports. Orthopaede 1995;24:252-67.

18. Thilmann AF, Schwart M, Töpper R et al. Different mechanisms underliw the long-latency stretch reflex response of active human muscle at different joints. J of Physiol 1991;444:631-43.

19. Frijns CJM, Laman DM, van Duijn MAJ et al. Normal values of patellar and ankle tendon reflex latencies. Clin Neurol Neurosur 1997;99:31-6.
20. Diener HC, Dichgans J, Bootz F, Bacher M. Early stabilization of human posture after a sudden disturbance: influence of rate and amplitude of displacement. Exp Brain Res 1984;56:126-34.

21. Atwood HL, MacKay WA. Neurophysiologie. Schattauer: 1994.

22. Cè E, Rampichini S, Monti E et al. Changes in the electromechanical delay components during a fatiguing stimulation in human skeletal muscle: an EMG, MMG and force combined approach. Eur J Appl Physiol 2017;117:95-117. DOI 10.1007/ s00421-016-3502-z

23. Cè E, Rampichini S, Limonta E, Esposito F. Reliability of the electromechanical delay components assessment during the relaxation phase. Physiology Journal 2013;517838:1-7.

24. Esposito F, Limonta E, Cè E. Passive stretching effects on electromechanical delay and time course of recovery in human skeletal muscle: new insights from an electromyographic and mechanomyographic combined approach. Eur J Appl Physiol 2011;111:485-95.

25. 25 Rothwell JC et al. Motor cortex stimulation in intact man. Brain 1987;110:1173-90.

26. Forgaard CJ, Franks IM, Maslovat D et al. Voluntary reaction time and long-latency reflex modulation. J Neurophysiol 2015;114:3386-99. DOI:10.1152/jn. 00648.2015.

27. Stam J, Tan KM. Tendon reflex variability and method of stimulation. Electroencephalography and clinical neurophysiology 1987;67:463-7.

28. Winter DA. Kinematic and kinetic patterns in human gait: Variability and compensating effects. Human Movement Science 1984;3:51-76.

29. Jelen P, Wit A, Dudzinski K, Nolan L. Expressing gait-line symmetry in able-bodied gait. Dynamic Medicine 2008;7:17. doi:10.1186/1476-5918-7-17

30. Bittmann F, Badtke G, Silbermann U. Kontrolle des Rehabilitationsverlaufes nach Knie- und Sprunggelenksverletzungen mit Hilfe der Computerdynographie. Poster presentation at the 34th congress of sports medicine 1995 in Saarbrücken, Germany. Deutsche Zeitschrift für Sportmedizin 1995;63:200.

31. Dickenson AH. Gate Control Theory of pain stands the test of time. Brit J Anaesthesia 2002;88:755-7.

Submitted: March 25, 2020

Revision received: April 8, 2020 Accepted for publication: April 9, 2020 